\title{
¿Qué es calidad de vida para un paciente con cáncer?
}

\section{For Patients Diagnosed with Cancer what is Qulity of Life? 0 que é qualidade de vida para um paciente com cancro?}

\author{
Ricardo Sánchez* \\ Universidad Nacional de Colombia \\ Fabio Alexander Sierra* \\ Fundación Universitaria de Ciencias de la Salud, Bogotá, Colombia \\ Elena Martín* \\ Instituto Nacional de Cancerología
}

Doi: dx.doi.org/10.12804/apl33.03.2015.01

\section{Resumen}

Objetivo: Evaluar el concepto de calidad de vida desde la perspectiva del paciente con cáncer. Método: Se evaluaron 1029 pacientes con cáncer de cuello uterino, mama, próstata, estómago, colon-recto, y un grupo de pacientes menores de edad con diferentes tipos de cáncer, efectuando una pregunta abierta (¿Cómo definiría usted la calidad de vida?). El texto de las respuestas fue procesado para realizar análisis lexicográfico y análisis de correspondencias. Resultados: Las formas gráficas y los segmentos repetidos más frecuentes se relacionaron con conceptos de bienestar, salud, funcionalidad, buena alimentación, aspectos familiares y espirituales. Estos conceptos varían según el tipo de cáncer. El análisis de correspondencias sugiere tres dimensiones: cuidado, bienestar y aspectos sociofamiliares y alimentarios. Hubo asociación entre estas dimensiones y el tipo de cáncer (dimensión 1: pacientes pediátricos; dimensión 2: cáncer de mama, y dimensión 3: pacientes con cáncer de próstata). En el espacio multidimensional el cáncer pediátrico se diferencia de las otras localizaciones de la enfermedad. Conclusiones: El diseño de instrumentos para medir calidad de vida en pacientes con cáncer debe incorporar particularidades relacionadas con el diagnóstico específico de la enfermedad.

Palabras clave: calidad de vida; neoplasias; formación de concepto.

\section{Abstract}

Objective: To evaluate the concept of quality of life from the patient's perspective. Method: 1029 patients having different diagnoses of cancer were evaluated using an open-ended question (How would you define Quality of Life?). The text of the individual replies was processed in order to perform lexicometric analysis which includes correspondence analysis. Results: Graphical forms and repeated segments that are most frequent are related to the concepts of well-being, health, functionality, good nutrition and spiritual and family aspects. These concepts change depending on type of cancer.

* Ricardo Sánchez, Facultad de Medicina, Universidad Nacional de Colombia, y Grupo de Investigación Clínica, Instituto Nacional de Cancerología; Fabio Alexander Sierra, División de investigaciones, Fundación Universitaria de Ciencias de la Salud; Elena Martín, Facultad de Medicina, Universidad Nacional de Colombia, y Grupo de Salud Mental, Instituto Nacional de Cancerología. La correspondencia relacionada con este artículo debe ser enviada a Ricardo Sánchez. Correo electrónico: rsanchezpe@unal.edu.co

Cómo citar este artículo: Sánchez, R., Sierra, F. A. \& Martín, E. (2015). ¿Qué es la calidad de vida para un paciente con cáncer? Avances en Psicología Latinoamericana, 33(3), 371-385. doi: dx.doi.org/10.12804/apl33.03.2015.01 
Correspondence analysis suggests three domains: one related to care, the other having to do with well-being and another one dealing with nutrition and social and family aspects. There was an association between these dimensions and type of cancer (dimension 1: pediatric patients; dimension 2: breast cancer; and dimension 3 : patients with prostrate cancer). In the multidimensional space that was constituted by graphical forms and repeated segments the diagnosis of pediatric cancer differs from others types of cancer. Conclusion: The design of instruments for measuring quality of live in patients having a cancer diagnosis should incorporate disease specific characteristics.

Keywords: quality of life; neoplasm; concept formation.

\section{Resumo}

Objetivo: Avaliar o conceito de qualidade de vida desde a perspectiva do paciente com cancro. Método: avaliaram-se 1029 pacientes com cancro cervical, mama, próstata, estômago, cólon-reto e um grupo de pacientes menores de idade com diferentes tipos de cancro, efetuando uma pergunta aberta (Como você definiria a qualidade de vida?). O texto das respostas foi processado para realizar análises lexicográfico e análises de correspondências. Resultados: as formas gráficas e os segmentos repetidos mais frequentes relacionaram-se com conceitos de bem-estar, saúde, funcionalidade, boa alimentação, aspetos familiares e espirituais. Estes conceitos variam segundo o tipo do cancro. A análise de correspondências sugere três dimensões: Cuidado - Bem-estar - Aspetos sociofamiliares e alimentares. Houve associação entre estas dimensões e o tipo de cancro (dimensão 1: pacientes pediátricos; dimensão 2: cancro de mama; e dimensão 3: pacientes com cancro de próstata). No espaço multidimensional, o cancro pediátrico diferencia-se das outras localizações da doença. Conclusões: a criação de instrumentos para medir qualidade de vida em pacientes com cancro deve incorporar particularidades relacionadas com o diagnóstico específico da doença.

Palavras-chave: qualidade de vida, neoplasias, formação de conceito.
La incorporación del concepto calidad de vida en el área de la salud es, de cierto modo, reciente y ha venido ganando importancia, en gran parte debido al cambio del paradigma para concebir la salud que tradicionalmente ponía el relieve en los desenlaces asociados con morbimortalidad. Este cambio de paradigma también se ha relacionado con modificaciones en el perfil epidemiológico, ya que el envejecimiento progresivo de la población se asocia con un incremento en la frecuencia de enfermedades crónicas y degenerativas en las cuales desenlaces como mortalidad pierden relevancia frente a aspectos como la calidad de vida (Bowling, 2005). Estos antecedentes llevan a que se plantee el concepto calidad de vida relacionada con la salud (CVRS), el cual se considera multidimensional, influenciado por la interacción de factores individuales, sociales y ambientales (Camfield \& Skevington, 2008), y claramente diferenciable por parte del paciente, de la percepción del estado de salud (Smith, Avis \& Assmann, 1999). En esta multidimensionalidad intervienen aspectos relacionados con, al menos, tres componentes: uno individual (relacionado con el estrés que supone la enfermedad para el paciente), uno de interacción (que tiene que ver con el apoyo social) y otro comunitaria (relacionado con un sentido de pertenencia) (Bramston, Chipuer \& Pretty, 2005).

A pesar de que la CVRS es un constructo que se evalúa cada vez con mayor frecuencia (Sánchez, Ballesteros \& Gómez, 2009), su conceptualización no es todavía clara: aunque la "calidad de vida", al igual que "la depresión" o "la histeria", por citar solo algunos, son conceptos usados en diferentes espacios (uno cotidiano y otro técnico en el área de la salud) con connotaciones que no necesariamente son coincidentes (Seidl \& Zannon, 2004), la calidad de vida se fundamenta en una experiencia personal y subjetiva, lo que acercaría las diferentes formas de conceptualización. Esta subjetividad se sugiere en estudios en los que se reporta que los médicos no detectan aspectos que para el paciente son importantes al evaluar su CVRS (Donovan, 1991), 
o en los que se encuentran discrepancias entre la calificación dada a la calidad de vida dependiendo si se hace por parte del personal de salud, del paciente o de sus familiares (Slevin, Plant, Lynch, Drinkwater \& Gregory, 1988).

También a favor de la subjetividad del constructo está el hallazgo de que factores incluidos en las escalas que miden CVRS, por ejemplo, el ambiente familiar, no necesariamente son vistos como importantes por todos los pacientes en estados terminales (Vig, Davenport \& Pearlman, 2002). También se ha visto que aspectos no incluidos habitualmente en los instrumentos diseñados para medir este constructo son relevantes para los pacientes con cáncer (O’Connell \& Skevington, 2005, 2010; Sánchez-Pedraza, Ballesteros \& Anzola, 2010). Uno de los que parecen más importantes en esta categoría de dimensiones olvidadas se relaciona con la espiritualidad y la religiosidad (O'Connell \& Skevington, 2005, 2007), y teniendo en cuenta la importancia que se ha venido encontrando en esta dimensión, se han diseñado instrumentos específicos para medir espiritualidad y religiosidad dentro del concepto global de calidad de vida (Skevington, Gunson \& O'Connell, 2013).

Además de la subjetividad del constructo, otro aspecto que se ha cuestionado sobre la medición de la CVRS es que el peso que se le asigna por parte de los pacientes a los diferentes dominios del constructo puede estar influenciado por factores como el grado de desarrollo del país en el que se vive (Molzahn, Kalfoss, Schick Makaroff \& Skevington, 2011) o por aspectos culturales que hacen que un instrumento desarrollado en un ambiente determinado presente propiedades psicométricas desfavorables cuando se valida en otro escenario cultural (Herdman, Fox-Rushby \& Badia, 1997; Skevington, 2002; Skevington, Sartorius \& Amir, 2004). Este aspecto se ha resaltado en el caso de instrumentos para medir calidad de vida en niños $\mathrm{y}$ adolescentes, en los que se ha cuestionado su estabilidad y se ha hecho hincapié en la necesidad de estudios transculturales que validen la utilidad de dichos instrumentos (Skevington, Dehner, Gi1lison, McGrath \& Lovell, 2014). Este fenómeno de falta de validez también se ha relacionado con falta de criterios definidos en los procesos de traducción que generan instrumentos sin equivalencia semántica entre los diferentes idiomas (Bowden \& Fox-Rushby, 2003).

Otros cuestionamientos que se han realizado a los instrumentos que miden CVRS es que están sesgados, pues incorporan los valores y las creencias de los que los diseñan, que no se aplican en la práctica clínica habitual del personal de salud y que quedan restringidos a escenarios de investigación que no necesariamente impactan la calidad de la atención brindada al paciente (Higginson \& Carr, 2001; Skevington, Day, Chisholm \& Trueman, 2005). Para manejar el inconveniente de la extrema subjetividad de la medición se han tratado de incorporar componentes ideográficos en los cuestionarios (Stenner, Cooper \& Skevington, 2003): por ejemplo, el Schedule for the Evaluation of Quality of Life (SEIQOL) (Joyce, McGee \& Oboyle, 1999); el Patient Generated Index (PGI) (Camilleri-Brennan, Ruta \& Steele, 2002; Ruta, Garratt, Leng, Russell \& MacDonald, 1994); el Global Person Generated Index (GPGI) (Camfield \& Ruta, 2007); el Índice de Salud de McMaster (Chambers, MacDonald, Tugwell, Buchanan \& Kraag, 1982), o el Subjective Quality of Life Profile (SQLP) (Dazord, 1997). Sin embargo, se ha planteado que la incorporación del componente ideográfico introduce dificultades en la interpretación y en el análisis de las encuestas con ese diseño y dificultan la estandarización requerida en casos como estudios multicéntricos o comparación del constructo entre diferentes culturas o países (Carr \& Higginson, 2001). Probablemente esta falta de un criterio unánime se ha reflejado en que, incluso en ensayos clínicos controlados que consideran como desenlace de importancia la CVRS, se hayan utilizado estrategias de medición que no tienen en cuenta las escalas diseñadas para medir este constructo (Sánchez et al., 2009). 
A pesar de las limitaciones que se han descrito, algunos autores defienden la utilización de instrumentos estandarizados para medir CVRS: para Testa (2000), la utilización de estos cuestionarios $\mathrm{y}$ escalas ha permitido monitorizar cambios en la condición del paciente, establecer pronósticos y definir patrones de referencia. Además, dichos instrumentos incorporan la multidimensionalidad del constructo: por ejemplo, en el caso de la medición de la calidad de vida en pacientes con cáncer el sistema FACIT considera cuatro dimensiones (Cella et al., 1993): estado físico general de salud, ambiente familiar, estado emocional y capacidad funcional. Por su parte, el sistema de EORTC (Aaronson, 1992; Aaronson et al., 1993) tiene en cuenta factores como síntomas físicos, funcionamiento cognoscitivo, desempeño funcional, componente emocional y factores sociales.

Teniendo en cuenta los anteriores planteamientos en los cuales se destaca la individualidad, la multidimensionalidad, la subjetividad y la relación con aspectos individuales, sociales y culturales en el constructo que se ha denominado CVRS, hemos realizado el presente estudio para establecer, en un grupo de pacientes con cáncer, cuáles son las principales dimensiones que se destacan según la opinión individual y particular del individuo enfermo en una situación potencialmente amenazante para su vida. Adicionalmente, nos interesa evaluar si estas dimensiones tienen alguna relación con diferentes tipos de cáncer. Para esto se empleará el análisis de correspondencias múltiples (ACM). Este es un tipo de análisis propuesto para evaluar relaciones entre variables categorizadas y entre niveles de estas variables (Costa, Santos, Cunha, Cotter \& Sousa, 2013). A diferencia de otras técnicas para el estudio de este tipo de variables, no se exige el cumplimiento de condiciones como un número mínimo de frecuencias esperadas o de valores perdidos (chi cuadrado, $z$ para proporciones, test exacto de Fisher). Una ventaja grande del ACM es que partiendo de la distancia euclídea entre los datos, se logra hacer una representación visual de las relaciones entre las variables en varias dimensiones (Costa et al., 2013). Por esto ha sido empleado en estudios que analizan relaciones entre factores psicosociales como la calidad de vida y variables sociodemográficas (Anota et al., 2014; Avolio et al., 2013; Skrzypczak, Czerniak \& Laski, 2012).

\section{Método}

\section{Población}

En el estudio se incluye un total de 1029 pacientes con diferentes tipos de cáncer confirmados histopatológicamente (cuello uterino, mama, próstata, estómago, colon-recto y un grupo de pacientes menores de edad con diagnóstico de cáncer).

\section{Procedimientos}

A los pacientes (o cuidadores de pacientes en el caso de menores de edad) se les efectuó la pregunta: ¿cómo define usted la calidad de vida? En el caso del grupo de menores de edad, esta se le formuló a su padre o al cuidador responsable. La selección de este tipo de diagnósticos se realizó considerando que estas patologías son los tipos más prevalentes en la población. Adicionalmente, se registró la edad y el sexo del paciente. Los datos se recolectaron entre los años 2010 y 2012 en los servicios de hospitalización y consulta del Instituto Nacional de Cancerología, en Bogotá, Colombia. Las respuestas dadas por los pacientes fueron transcritas al pie de la letra y se utilizaron para realizar un análisis lexicográfico.

\section{Análisis estadístico}

Para el análisis lexicográfico se tomó como insumo las formas gráficas (sucesión de caracteres comprendidos entre dos delimitadores que pueden ser espacios o signos de puntuación). El vocabulario recopilado (es decir, el conjunto de formas 
gráficas) tuvo un preprocesamiento en el que se efectuaron los siguientes pasos:

1. Síntesis de formas gráficas: este paso implicó seleccionar una sola forma gráfica para términos que fueran sinónimos (por ejemplo casa reemplaza las formas apartamento, casita, rancho, residencia).

2. Delimitación del vocabulario para reducir la redundancia. Este paso supone los siguientes procedimientos:

- Eliminación de palabras herramientas que corresponden a artículos, conjunciones, preposiciones y pronombres.

- Selección de un umbral de frecuencia de aparición de formas gráficas. En este caso se eliminaron del vocabulario las formas que tuvieran una frecuencia de aparición menor que tres.

La siguiente etapa del análisis consistió en establecer las respuestas características (RC): estas son respuestas, tal como las dan los pacientes, que son representativas del texto en su conjunto, o del texto agrupado en categorías. Las RC se ubicaron con base en el criterio del valor medio, el cual se obtiene calculando las medias de los valores test de las formas características que las conforman. De esta manera se seleccionaron las formas características con valores test medios mayores que 1.96.

Tomando como insumo las formas gráficas simples (palabras sueltas) y los segmentos repetidos detectados en el corpus (combinación de formas gráficas que se presentan de manera repetida) se realizó un análisis de correspondencias, en el cual se utilizaron estas formas y segmentos repetidos como modalidades activas, y la agrupación de los seis tipos de cáncer y el sexo (masculino-femenino) como modalidades ilustrativas. La determinación del número de dimensiones se dio a partir de los cambios de inercia de un diagrama de sedimenta- ción de los valores propios. Para escoger las formas que representan mejor cada uno de los ejes de la dimensión reducida se tomaron como criterios los valores de contribución al eje y los cosenos cuadrados. Los valores de contribución indican qué proporción de la inercia total sobre un eje en una determinada dirección es debida a una modalidad (se reporta como valores test sobre una distribución normal estandarizada) y el coseno cuadrado muestra que parte de la inercia total de una modalidad se recoge en una dirección particular del eje. Los procedimientos de análisis estadístico se efectuaron con el programa SPAD 5®. El estudio fue aprobado y vigilado por el Comité de Ética en Investigaciones del Instituto Nacional de Cancerología.

\section{Resultados}

Las respuestas correspondieron a pacientes con seis diferentes tipos de cáncer: mama $(N=$ $114 ; 11.08 \%)$, cuello uterino $(N=202 ; 19.63 \%)$, estómago $(N=200 ; 19.44 \%)$, próstata $(N=111$; $10.79 \%)$, colon-recto $(N=201 ; 19.53 \%)$ y 201 casos correspondientes a distintos tipos de cáncer en menores de edad (19.53\%). La edad tuvo una media de 48.64 años ( $D E=22.9$ años); en el grupo de pacientes adultos la edad tuvo un media de 58 años $(D E=13.7$ años) y en el de menores de edad 10 años $(D E=5.9$ años). De acuerdo con la localización del cáncer, las medias de la edad fueron las siguientes: mama: $56.7(D E=11.05)$; cuello uterino: $53.24(D E=12.43)$; estómago: $59.23(D E$ $=16.84)$; próstata $66.5(D E=7.25)$, y colon-recto: $57.9(D E=13.2)$. Un $56.2 \%$ de los pacientes $(N=$ 578) fueron de sexo femenino.

Se obtuvieron 1029 respuestas que contuvieron 18682 palabras (este total representa el tamaño del vocabulario). En el total del vocabulario se encontraron 1822 palabras diferentes, lo que supone una riqueza del vocabulario del 9.8\%.

Las formas gráficas que tuvieron mayor frecuencia de aparición se relacionaron con bienestar, salud, funcionalidad, alimentación, familia y aspec- 
tos espirituales (tabla 1). Los segmentos repetidos (combinaciones de formas gráficas que se presentan de manera constante en el corpus) muestran una tendencia similar a la de las formas gráficas, pero aparecen adicionalmente conceptos relacionados con lo emocional y el sueño (tabla 2).

Tabla 1

Formas gráficas y frecuencia de aparición en el corpus

\begin{tabular}{cc}
\hline Palabras empleadas & Frecuencia \\
\hline tener & 852 \\
bien & 678 \\
estar & 654 \\
salud & 585 \\
familia & 282 \\
buena & 248 \\
vivir & 243 \\
poder & 220 \\
vida & 219 \\
todo & 128 \\
dios & 112 \\
comer & 103 \\
hijos & 102 \\
\hline
\end{tabular}

En relación con las respuestas características, el concepto de salud ocupa un lugar predominante en pacientes con cáncer de próstata, estómago y en el grupo de pacientes pediátricos. Salud, tranquilidad emocional y económica y acompañamiento familiar son los aspectos relevantes para los pacientes con cáncer de próstata (hombres). Para las pacientes con cáncer de cuello uterino o mama (mujeres), los conceptos centrales se relacionan con el ambiente familiar, la disponibilidad de tratamiento y aspectos espirituales (tabla 3 ). Dentro de las respuestas características de pacientes con cáncer digestivo (tabla 4) se destaca que en los pacientes con cáncer colorrectal se hace hincapié en el mantenimiento de la capacidad laboral; mientras que en el grupo de pacientes con cáncer
Tabla 2

Segmentos repetidos más frecuentes

\begin{tabular}{|c|c|}
\hline Texto del segmento & Frecuencia \\
\hline estar bien & 272 \\
\hline tener salud & 148 \\
\hline buena salud & 98 \\
\hline vivir bien & 87 \\
\hline tener buena & 87 \\
\hline comer bien & 62 \\
\hline tener buena salud & 48 \\
\hline sentirme bien & 48 \\
\hline salud estar & 44 \\
\hline estar alentado & 44 \\
\hline buena alimentación & 42 \\
\hline poder trabajar & 38 \\
\hline bienestar & 33 \\
\hline tener trabajo & 32 \\
\hline tener tranquilidad & 30 \\
\hline dormir bien & 28 \\
\hline poder comer & 25 \\
\hline buena relación & 21 \\
\hline poder hacer & 20 \\
\hline familia tener & 20 \\
\hline bien dormir & 19 \\
\hline alimentarme bien & 18 \\
\hline estar tranquilo & 18 \\
\hline tener salud estar & 17 \\
\hline poder disfrutar & 17 \\
\hline estar contento & 17 \\
\hline tener todo & 16 \\
\hline tener problemas & 15 \\
\hline comer bien dormir & 15 \\
\hline estar tranquila & 15 \\
\hline
\end{tabular}

gástrico se reportan múltiples aspectos, similares a los reportados por los pacientes con cáncer de próstata (salud, tranquilidad emocional y económica 
y acompañamiento familiar). En el grupo de pacientes pediátricos se incorporan cuestiones relacionadas con el acceso a los sistemas de salud, la educación, los aspectos afectivos y cubrimiento de necesidades básicas (tabla 5).

Tabla 3

Respuestas características de pacientes con cáncer de próstata, cuello uterino y mama

\begin{tabular}{|c|c|c|}
\hline & $\begin{array}{l}\text { Respuestas características pacientes cáncer } \\
\text { de próstata }\end{array}$ & $\begin{array}{c}\text { Valor } \\
\text { test }\end{array}$ \\
\hline 1 & $\begin{array}{l}\text { "tener salud, tener tranquilidad, poderse mo- } \\
\text { ver, tener actividad". }\end{array}$ & 2.576 \\
\hline 2 & $\begin{array}{l}\text { "tener salud, tener cómo solventar el sus- } \\
\text { tento diario, tener recreación, tener buenos } \\
\text { amigos y tener buenas relaciones con las } \\
\text { demás personas". }\end{array}$ & 2.411 \\
\hline 3 & $\begin{array}{l}\text { "vivir en paz interior. No tener dolores; po- } \\
\text { der movilizarse uno; tener buenas relaciones } \\
\text { con los demás en cuanto a bienestar, alegría. } \\
\text { Es tener una actitud positiva ante la vida" }\end{array}$ & 2.381 \\
\hline 4 & $\begin{array}{l}\text { "tener salud es lo principal; tener armonía } \\
\text { familiar; tener los medios económicos para } \\
\text { tener una buena dieta no solo con harinas. } \\
\text { No tener deudas o tener cómo responder por } \\
\text { ellas. Tener vida sexual como antes". }\end{array}$ & 2.369 \\
\hline 5 & $\begin{array}{l}\text { "tener buena memoria; dormir bien, comer } \\
\text { bien, tener buenas condiciones de vida; tener } \\
\text { un buen ambiente familiar; vivir contento; } \\
\text { no tener vicios". }\end{array}$ & 2.359 \\
\hline 6 & $\begin{array}{l}\text { "es un bienestar personal y familiar; y la } \\
\text { correspondencia de ellos; tener estatus si no } \\
\text { boyante, por lo menos pasable; tener tranqui- } \\
\text { lidad incluida la económica". }\end{array}$ & 2.302 \\
\hline 7 & $\begin{array}{l}\text { "vivir bien, no tener problemas, tener la co- } \\
\text { mida, tener armonía con los familiares, con } \\
\text { los que uno vive" }\end{array}$ & 2.298 \\
\hline 8 & "llevar tranquilidad, tener una vida tranquila". & 2.291 \\
\hline 9 & $\begin{array}{l}\text { "vivir bien; tener salud; tener facilidades } \\
\text { económicas, no tener estrés". }\end{array}$ & 2.249 \\
\hline 10 & $\begin{array}{l}\text { "es vivir bien, dormir bien, comer bien, tener } \\
\text { tranquilidad. No tener disgustos, ni rencores". }\end{array}$ & 2.248 \\
\hline 11 & $\begin{array}{l}\text { "sentirse uno con capacidad para trabajar. } \\
\text { Poder comer y tener con qué comer; comer } \\
\text { bien. Tener un buen apartamento. Tener } \\
\text { carro, tener comodidades. Dormir bien, no } \\
\text { tener dolores". }\end{array}$ & 2.246 \\
\hline
\end{tabular}

"es el conjunto de las cualidades o condiciones que permiten que uno pueda salir a

12 pasear, no tener dolores, tener el apoyo de la familia de los amigos. Tener tranquilidad, no tener preocupaciones".

"estar con salud completa y no tener ningún

13 dolor. Tener buenas relaciones con los demás y vivir contento, tener asistencia médica frecuente para que los males no tomen ventaja”.

Respuestas características pacientes cáncer de cuello uterino

Valor test

1 "pues estar uno visitando al médico, estar en los exámenes".

"estar consultando con los médicos, estar

2 bien con mis hijos, con mis nietos, todos los que nos rodean".

"yo soy madre cabeza de hogar, de mi dependen, tengo uno mayor de edad, y tengo tres en bachillerato, y nietos que dependen

3 de mí. No soy de acá, y entonces ahora tengo que ver qué hacer para estar bien, si me van a hacer una cirugía yo tendré que ver la forma de traerlos, para que estén conmigo, porque ellos dependen de mí”.

"es de pronto la calidad de vida que nosotros llevamos, cómo debemos llevarlos, cuidar-

4 nos, asistir a las citas que nos coloquen, asis- 2.104 tir a los controles, exámenes para así saber cómo está uno del estado de salud".

“yo entiendo que es la riqueza más grande,

5 que es la salud, es estar con mis hijos. Dios es muy importante para mi vida, y en mi calidad de vida ya que es el que me da todo".

"pues la calidad de vida, cuidarnos, hacernos todos los tratamientos como nos indican los médicos, asistir a todas las citas médicas y alimentarnos bien".

"tener salud, estar con mis hijos, tener

7 trabajo, que mis hijos estén alentados, y la alimentación".

"para mi calidad de vida es estar con mis hi-

8 jos, que tenga un ranchito y la salud, y tener a dios y a mi nieto junto a mí".

"que me atiendan, que mi familia, que la institución estén pendientes de mi enfermedad, que me acompañen, no estar sola, estar con la gente que me quiere, con mis hijos". 


\begin{tabular}{llc}
\hline Respuestas características cáncer de mama & $\begin{array}{c}\text { Valor } \\
\text { test }\end{array}$ \\
\hline 1 "el tiempo en el tratamiento que ha sido mara- & 2.399 \\
& $\begin{array}{l}\text { villoso, el tratamiento ha mejorado mi vida". } \\
\text { "disfrutar de lo que tengo con amor para que }\end{array}$ \\
2 así sea más llevadera mi permanencia en mis & 2.065 \\
& $\begin{array}{l}\text { tratamientos". } \\
3\end{array}$ "verse una persona normal y sin enfermedades". & 1.985 \\
\hline
\end{tabular}

Tabla 4

Respuestas características de pacientes con cáncer del tubo digestivo

\begin{tabular}{|c|c|c|}
\hline & Respuestas características cáncer colon-recto & $\begin{array}{l}\text { Valor } \\
\text { test }\end{array}$ \\
\hline 1 & "trabajar, poder trabajar y ser feliz". & 2.391 \\
\hline 2 & "no preocuparse demasiado, poder trabajar". & 1.967 \\
\hline \multirow[t]{2}{*}{3} & $\begin{array}{l}\text { "estar bien, que me curen, poder trabajar, } \\
\text { poder producir". }\end{array}$ & 1.961 \\
\hline & Respuestas características cáncer gástrico & $\begin{array}{l}\text { Valor } \\
\text { test }\end{array}$ \\
\hline 1 & "tener salud y estar bien con la familia". & 3.422 \\
\hline 2 & $\begin{array}{l}\text { "estar bien económicamente y estar bien con } \\
\text { la familia". }\end{array}$ & 3.242 \\
\hline 3 & $\begin{array}{l}\text { "estar bien de salud, con su familia, tener } \\
\text { trabajo". }\end{array}$ & 3.131 \\
\hline 4 & $\begin{array}{l}\text { "tener salud, estar con la familia y estar bien } \\
\text { con Dios". }\end{array}$ & 3.072 \\
\hline 5 & $\begin{array}{l}\text { "es estar bien de salud, estar con la familia, } \\
\text { la esposa, los hijos, tener trabajo". }\end{array}$ & 2.856 \\
\hline 6 & $\begin{array}{l}\text { "tener buena alimentación, estar contenta, } \\
\text { estar con la familia, tener salud". }\end{array}$ & 2.744 \\
\hline 7 & "estar bien económicamente. Salud". & 2.739 \\
\hline 8 & $\begin{array}{l}\text { "estar bien de salud, económicamente, estar } \\
\text { bien con la familia, estar acompañado, tener } \\
\text { su droguita". }\end{array}$ & 2.727 \\
\hline 9 & $\begin{array}{l}\text { "tener salud, tranquilidad, tener para comer, } \\
\text { estar bien económicamente". }\end{array}$ & 2.697 \\
\hline 10 & $\begin{array}{l}\text { "la salud, estar acompañado de mi familia, } \\
\text { estar en la casa alentado". }\end{array}$ & 2.675 \\
\hline 11 & $\begin{array}{l}\text { "que estuviera la familia reunida, tener bue- } \\
\text { na salud, estar bien económicamente". }\end{array}$ & 2.645 \\
\hline 12 & “estar aliviado, poder comer”. & 2.581 \\
\hline 13 & $\begin{array}{l}\text { "vivir en tranquilidad con la familia, salud, y } \\
\text { poder viajar". }\end{array}$ & 2.533 \\
\hline
\end{tabular}

"tener uno lo mejor, tener mejor servicio en

14 salud, estar bien, estar tranquila, estar apoya- 2.486 da de mi familia".

15 "es estar uno bien, estar con la familia, tener trabajo, tener ánimo para todo”.

"estar alentado y tener las cosas de su ranchito".

"estar bien de salud, estar bien contento con la familia y poder compartir así sea un dulce”.

18 "estar bien, poder disfrutar, que no le duela a

18 uno nada, poder comer, poder estar activa".

Tabla 5

Respuestas características de pacientes del grupo de cáncer en población pediátrica

\begin{tabular}{|c|c|c|}
\hline & $\begin{array}{c}\text { Respuestas características cáncer grupo } \\
\text { pediátrico }\end{array}$ & $\begin{array}{l}\text { Valor } \\
\text { test }\end{array}$ \\
\hline 1 & “estudio y educación”. & 4.963 \\
\hline 2 & "atención, salud y amor" & 4.13 \\
\hline 3 & "salud y buena educación”. & 3.932 \\
\hline 4 & $\begin{array}{l}\text { "atención oportuna en todos los aspectos y } \\
\text { cariño". }\end{array}$ & 3.781 \\
\hline 5 & "buen servicio y buena atención". & 3.542 \\
\hline 6 & $\begin{array}{l}\text { "obtener todo lo que se sueña y sobrepasar } \\
\text { los obstáculos". }\end{array}$ & 3.542 \\
\hline 7 & "salud completa, educación, apoyo y amor". & 3.507 \\
\hline 8 & $\begin{array}{l}\text { "el buen alimento, la educación, vivienda } \\
\text { digna, comodidad y buena atención". }\end{array}$ & 3.446 \\
\hline 9 & $\begin{array}{l}\text { "buen estatus económico, apoyo incondicio- } \\
\text { nal y una buena y oportuna salud". }\end{array}$ & 3.368 \\
\hline 10 & "alimentación, salud y estudio". & 3.088 \\
\hline 11 & $\begin{array}{l}\text { "alimentación, educación, buen ejemplo y } \\
\text { sobre todo salud". }\end{array}$ & 3.08 \\
\hline 12 & $\begin{array}{l}\text { "buena vivienda, buena comida, buen trato y } \\
\text { oportunidades de superación". }\end{array}$ & 2.986 \\
\hline 13 & "salud, buen trato, respeto y servicio". & 2.957 \\
\hline 14 & $\begin{array}{l}\text { "salud, educación, alimentación. Que todo } \\
\text { funcione bien". }\end{array}$ & 2.951 \\
\hline 15 & $\begin{array}{l}\text { "atención de salud, su tratamiento. Que ten- } \\
\text { ga toda la atención que se merece". }\end{array}$ & 2.846 \\
\hline 16 & $\begin{array}{l}\text { "mejorar, salud, alimentación, vestido y } \\
\text { vivienda". }\end{array}$ & 2.703 \\
\hline
\end{tabular}




\begin{tabular}{|c|c|c|}
\hline & $\begin{array}{c}\text { Respuestas características cáncer grupo } \\
\text { pediátrico }\end{array}$ & $\begin{array}{c}\text { Valor } \\
\text { test }\end{array}$ \\
\hline 17 & "el buen trato que le den y la buena salud". & 2.644 \\
\hline 18 & $\begin{array}{l}\text { "el acceso oportuno a los servicios de salud, } \\
\text { alimentación y cariño" }\end{array}$ & 2.616 \\
\hline 19 & "bienestar, protección y apoyo". & 2.6 \\
\hline 20 & $\begin{array}{l}\text { "estudio, alimentación, buen trato, recrea- } \\
\text { ción, actividades lúdicas, acompañamiento } \\
\text { en salud". }\end{array}$ & 2.555 \\
\hline 21 & $\begin{array}{l}\text { "contar con lo básico para vivir contento y } \\
\text { feliz". }\end{array}$ & 2.515 \\
\hline 22 & $\begin{array}{l}\text { "contar con los recursos necesarios para cu- } \\
\text { brir necesidades básicas". }\end{array}$ & 2.475 \\
\hline 23 & $\begin{array}{l}\text { "atención en salud buena y eficaz, estudio y } \\
\text { tener lo esencial para vivir". }\end{array}$ & 2.455 \\
\hline 24 & $\begin{array}{l}\text { "garantía de contar con los servicios necesa- } \\
\text { rios para el bienestar en general". }\end{array}$ & 2.425 \\
\hline 25 & $\begin{array}{l}\text { "tener atención oportuna, cariño y acompa- } \\
\text { ñamiento". }\end{array}$ & 2.412 \\
\hline
\end{tabular}

El análisis de correspondencias se efectuó sobre un espacio con tres dimensiones cuya estructura se muestra en la tabla 6 . El eje 1 agrupa en uno de los polos conceptos relacionados con el cuidado (educación, amor, buen trato, necesidades básicas, atención en salud), y en el otro, aspectos familiares (familia e hijos), de tal manera puede plantearse que este eje 1 engloba aspectos relacionados con el cuidado en el ambiente familiar. Dentro de las variables ilustrativas, la modalidad mejor representada en dicho eje corresponde al grupo de pacientes pediátricos (sus coordenadas son 65 en el polo negativo y cuatro en el positivo). El eje 2 es una dimensión relacionada fundamentalmente con el bienestar; solo agrupa modalidades en el polo negativo (tabla 6). En este eje, la modalidad mejor representada corresponde al grupo de pacientes con cáncer de mama (coordenada de 51 en el polo negativo y de cinco en el positivo). Finalmente, el eje 3 representa una dimensión de lo sociofamiliar y alimentario: agrupa en el polo negativo aspectos relacionados con la vida y los hijos y en el positivo el tener buenas relaciones y poder alimentarse; en este eje, la modalidad que mejor queda representada es la del grupo de pacientes con cáncer de próstata (sus coordenadas son 48 en el eje negativo y nueve en el positivo).

Tabla 6

Conformación de los ejes según el análisis de correspondencias binarias

\begin{tabular}{lclc}
\multicolumn{4}{c}{$\begin{array}{c}\text { Elementos activos de mayor contribución a la formación } \\
\text { del eje } 1 \text { (valor test }>1.96)\end{array}$} \\
\hline \multicolumn{3}{c}{ Zona negativa } & \multicolumn{3}{c}{ Zona positiva } \\
\hline necesidad básicas & 1.9 & familia & 1.9 \\
atención oportuna & 1.9 & hijos & 1.9 \\
mucho amor & 1.9 & \\
buen trato & 2 & \\
buena vida & 2 & \\
amor & 3.5 & 3.8
\end{tabular}

Elementos activos de mayor contribución a la formación del eje 2 (valor test $>1.96$ )

\begin{tabular}{lll}
\hline \multicolumn{2}{c}{ Zona negativa } & Zona positiva \\
\hline bien sentirme bien & 1.9 & \\
bien sentirme & 2.2 & \\
sentirme bien & 3.6 & \\
\hline
\end{tabular}

Elementos activos de mayor contribución a la formación del eje 3 (valor test $>1.96$ )

\begin{tabular}{lclc}
\hline \multicolumn{2}{c}{ Zona negativa } & \multicolumn{2}{c}{ Zona positiva } \\
\hline vida & 1.9 & tener buenas & 1.9 \\
hijos & 2.2 & tener buenas relaciones & 2 \\
& & poder comer & 2.3 \\
\hline
\end{tabular}

La representación de las modalidades ilustrativas en los espacios factoriales se muestra en las figuras 1 y 2 . Puede verse que las modalidades de la variable sexo se aproximan al centro de los ejes; mientras que las modalidades correspondientes al grupo de pacientes pediátricos y al de cáncer de mama (especialmente el primero) tienden a relacionarse poco con los otros tipos de diagnóstico. 


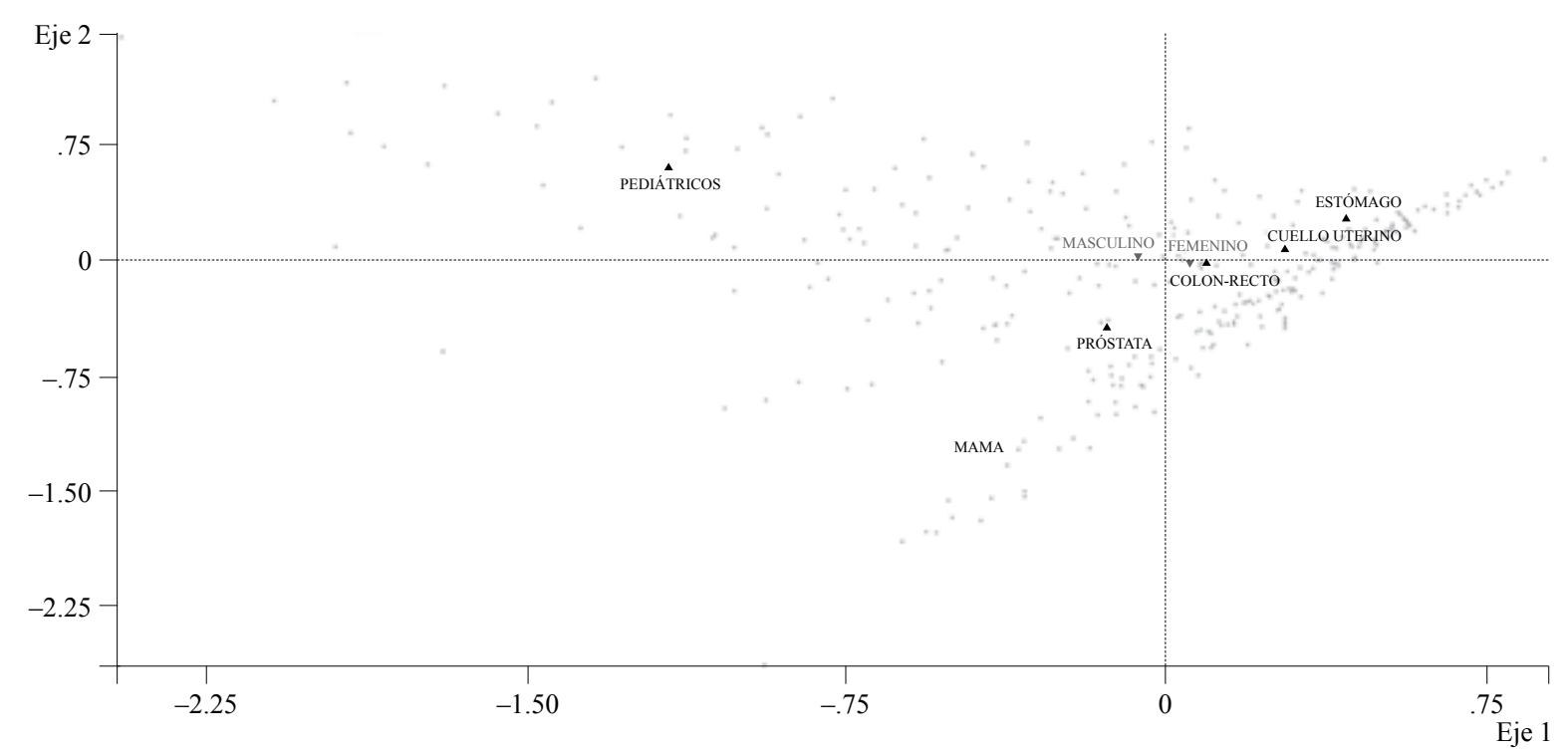

Figura 1. Ubicación de las variables ilustrativas sobre los ejes 1 y 2

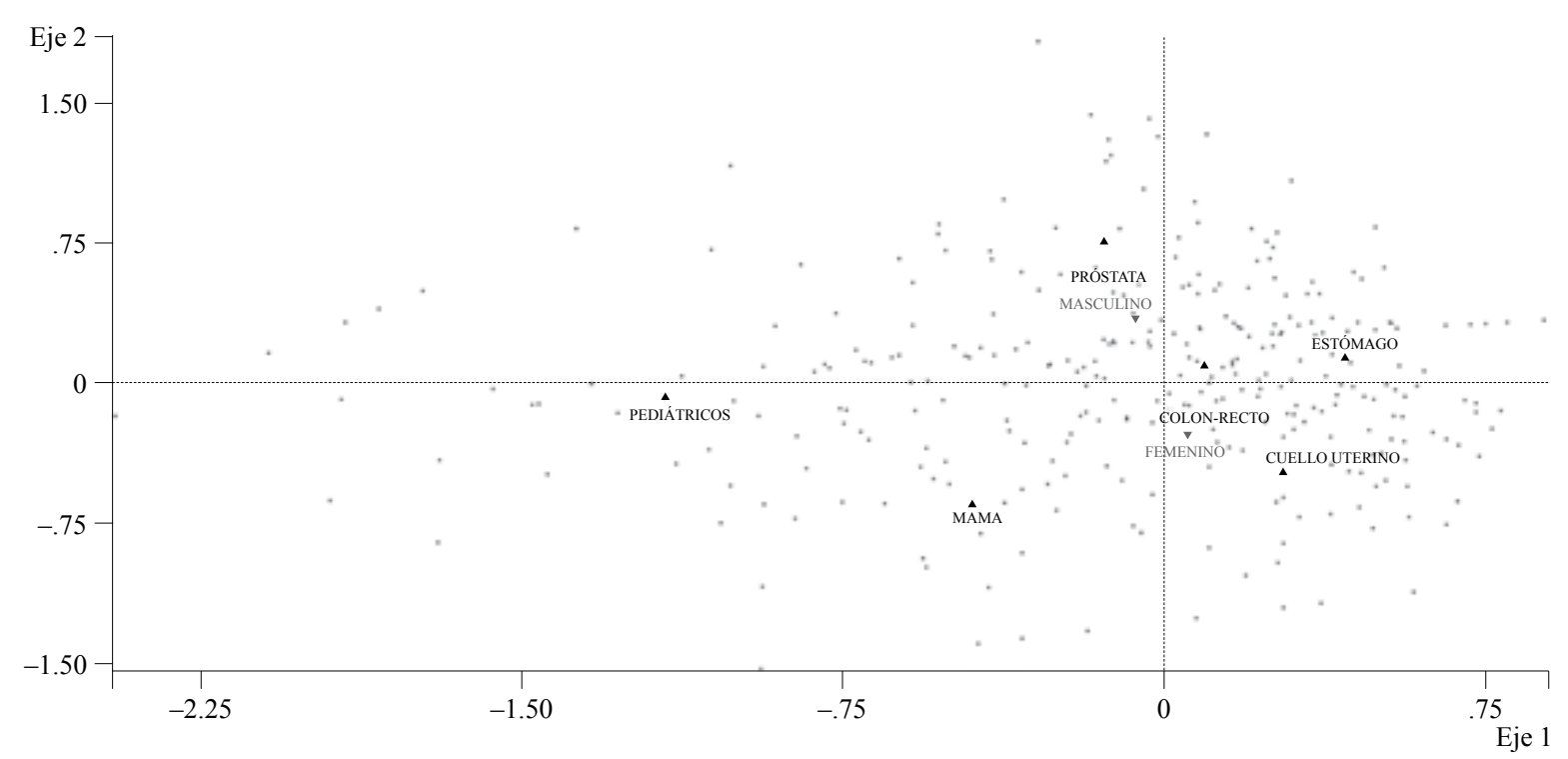

Figura 2. Ubicación de las variables ilustrativas sobre los ejes 1 y 3

\section{Discusión}

En el presente estudio se incluyeron pacientes con los tipos de cáncer de mayor frecuencia en el mundo (International Agency for Research on Cancer \& World Health Organization, 2008) y, adicionalmente, se tuvo en cuenta una categoría heterogénea, que poco se ha considerado en otros estudios sobre este tema, relacionada con el cáncer en población pediátrica.

Al indagar sobre un concepto general de calidad de vida en este grupo de pacientes o cuidadores de pacientes con cáncer, se destaca que la salud y el bienestar son aspectos centrales en esta dimensión; esto ha sido reconocido en diferentes estudios en los que se considera que el estado de salud es uno 
de los más importantes determinantes de la calidad de vida general (Seidl y Zannon, 2004). Otros componentes que se evidencian en los conceptos de esta muestra de pacientes, como la funcionalidad, los aspectos familiares y la espiritualidad, han sido incorporados en diferentes instrumentos que evalúan el constructo denominado CVRS (Aaronson et al., 1993; Cella et al., 1993). El componente relacionado con el acceso a los sistemas de salud, que se encontró asociado a algunos tipos de cáncer, ya había sido reportado en un estudio similar realizado en Colombia y podría estar reflejando características particulares del sistema de salud que existe en este país.

La CVRS se ha medido con instrumentos generales o específicos. Los instrumentos generales permiten comparar diferentes condiciones e intervenciones, pero no se enfocan adecuadamente en el área de impacto de una intervención particular. Los instrumentos específicos tienen una mayor capacidad de responder a los cambios o se enfocan en subgrupos precisos de pacientes, pero tienen la limitación de no permitir comparaciones entre diferentes patologías. Estos instrumentos están diseñados para evaluar estados específicos de alguna enfermedad o áreas particulares de funcionamiento, como pueden ser los diferentes tipos de cáncer (Bowling, 2005).

Los resultados del análisis de las respuestas características podría estar a favor del uso de instrumentos específicos, pues los hallazgos sugieren que el concepto de calidad de vida puede estar relacionado con el tipo de cáncer que presenta el paciente: de esta manera, se destaca en el grupo de pacientes con cáncer de cuello uterino o de mama la preocupación por aspectos espirituales y por la disponibilidad del tratamiento; en los pacientes con cáncer colorrectal, el mantenimiento de la capacidad laboral, y en los cuidadores de menores de edad con cáncer, aspectos relacionados con educación y con la satisfacción de las necesidades básicas.

Los hallazgos en el grupo de pacientes con cáncer de mama o cuello uterino concuerdan con un estudio previo en mujeres con cáncer que utilizó esta misma metodología de análisis (Sánchez-Pedraza et al., 2010). Sin embargo, las subescalas que incorporan instrumentos específicos de uso habitual para medir calidad de vida en cáncer (EORTC BR29 y FACIT B, para cáncer de mama; FACIT-Cx y EORTC CX24 para cáncer de cuello uterino; EORTC pr25 y FACIT-P, para cáncer de próstata; EORT STO-22 y FACIT-Ga, para cáncer gástrico, y EORTC CR-29 y FACIT-C para cáncer colorrectal) destacan el componente de síntomas de la enfermedad o los relacionados con el tratamiento, pero no aspectos relacionados con acceso a tratamiento, espiritualidad, educación o satisfacción de necesidades básicas, que son dimensiones consideradas importantes por esta muestra de pacientes.

Los resultados del análisis de correspondencias sugieren que las agrupaciones de conceptos asociados con calidad de vida se relacionan más con la localización del cáncer o de la población afectada por la enfermedad (adultos-niños) que por el sexo. En relación con el tipo de población, se encontró que las agrupaciones de conceptos son diferentes en pacientes adultos que en cuidadores de pacientes pediátricos. También se encuentra que en esta muestra de pacientes el concepto de calidad de vida se encuentra representado por tres dimensiones (componente relacionado con el cuidado, bienestar y componente sociofamiliar-alimentario). En cada una de estas, las variables ilustrativas se proyectan de manera específica, lo cual refuerza el planteamiento previo en relación con el tipo de cáncer y la presencia de conceptos específicos sobre calidad de vida. Llama la atención que para los cuidadores de menores de edad con cáncer los conceptos de calidad de vida se relacionan con aspectos que están vinculados a las prácticas de crianza (cubrir necesidades básicas, amor, educación, cuidado).

Las escalas específicas podrían incorporar la medición de aspectos más generales que podrían estar relacionados con el tipo de cáncer.

Las controversias sobre el concepto de calidad de vida y sobre las estrategias que se han empleado 
para su medición deben reflejarse en el desarrollo de técnicas de evaluación que permitan disponer de herramientas y estrategias que redunden en un mayor bienestar del paciente y, eventualmente, en el desarrollo y aplicación de técnicas de medición que permitan incorporar de manera válida y confiable los aspectos de subjetividad y heterogeneidad que caracterizan este constructo. Esto también supone que la aplicación de estas técnicas en contextos sociales y culturales diferentes de aquellos donde se desarrolló un instrumento particular se haga luego de procesos juiciosos de validación; en este sentido, vale la pena mencionar que en Colombia es escasa la disponibilidad de instrumentos para medir calidad de vida que hayan sido sometidos a validaciones rigurosas.

Como limitaciones del presente estudio somos conscientes de que no se incluyeron otras fuentes de variabilidad como la escolaridad, el nivel socioeconómico, el tiempo de evolución o el estadio de la enfermedad (sin embargo algunos estudios sugieren que el estadio no es un marcador fuerte de calidad de vida (Sánchez, Alexander-Sierra \& Oliveros, 2012). Por ejemplo, se ha encontrado que la calidad de vida en niños se relaciona con aspectos como el sexo (es mayor en niñas) y el nivel educativo y de ingreso de los padres (Jirojanakul, Skevington \& Hudson, 2003). El no haber indagado sobre el concepto de calidad de vida directamente en los pacientes pediátricos puede ser una limitación parcial del estudio, dado que el tipo de exploración se hacía sobre un aspecto subjetivo, que la población pediátrica fue muy joven y que hay reportes que sugieren que la exploración de conceptos subjetivos directamente en niños de poca edad puede tener problemas de validez (Jozefiak, Larsson, Wichstrom, Wallander \& Mattejat, 2010; Wallander, Schmitt \& Koot, 2001).

\section{Referencias}

Aaronson, N. K. (1992). Assessing the quality of life of patients in cancer clinical trials: Com- mon problems and common sense solutions. European Journal of Cancer, 28A(8-9), 1304-1307.

Aaronson, N. K., Ahmedzai, S., Bergman, B., Bullinger, M., Cull, A., Duez, N. J., . . . et al. (1993). The European Organization for Research and Treatment of Cancer QLQ-C30: a quality-of-life instrument for use in international clinical trials in oncology. Journal of the National Cancer Institute, 85(5), 365-376.

Anota, A., Bascoul-Mollevi, C., Conroy, T., Guillemin, F., Velten, M., Jolly, D., . . . Bonnetain, F. (2014). Item response theory and factor analysis as a mean to characterize occurrence of response shift in a longitudinal quality of life study in breast cancer patients. Health and Quality of Life Outcomes, 12(1), 32. doi: 10.1186/14777525-12-32

Avolio, M., Montagnoli, S., Marino, M., Basso, D., Furia, G., Ricciardi, W., \& de Belvis, A. G. (2013). Factors influencing quality of life for disabled and nondisabled elderly population: the results of a multiple correspondence analysis. Current Gerontology and Geriatrics Research, 258274. doi: 10.1155/2013/258274

Bowden, A., \& Fox-Rushby, J. A. (2003). A systematic and critical review of the process of translation and adaptation of generic health-related quality of life measures in Africa, Asia, Eastern Europe, the Middle East, South America. Social Science \& Medicine, 57(7), 1289-1306.

Bowling, A. (2005). Measuring health: A review of quality of life measurement scales (3rd ed.). Maidenhead, Berkshire, England: Open University Press.

Bramston, P., Chipuer, H., \& Pretty, G. (2005). Conceptual principles of quality of life: An empirical exploration. Journal of Intellectual Disability Research, 49(Pt 10), 728-733.

Camfield, L., \& Ruta, D. (2007). "Translation is not enough": using the Global Person Generated Index (GPGI) to assess individual quality of life in Bangladesh, Thailand, and Ethiopia. Quality 
of Life Research: An International Journal of Quality of Life Aspects of Treatment, Care and Rehabilitation, 16(6), 1039-1051. doi: 10.1007/ s11136-007-9182-8

Camfield, L., \& Skevington, S. M. (2008). On subjective well-being and quality of life. Journal of Health Psychology, 13(6), 764-775. doi: 10.1177/1359105308093860

Camilleri-Brennan, J., Ruta, D. A., \& Steele, R. J. (2002). Patient generated index: New instrument for measuring quality of life in patients with rectal cancer. World Journal of Surgery, 26(11), 1354-1359. doi: 10.1007/s00268-0026360-2

Carr, A. J., \& Higginson, I. J. (2001). Are quality of life measures patient centred? British Medical Journal, 322(7298), 1357-1360.

Cella, D. F., Tulsky, D. S., Gray, G., Sarafian, B., Linn, E., Bonomi, A., ... et al. (1993). The Functional Assessment of Cancer Therapy scale: Development and validation of the general measure. Journal of Clinical Oncology: Official Journal of the American Society of Clinical Oncology, 11(3), 570-579.

Chambers, L. W., Macdonald, L. A., Tugwell, P., Buchanan, W. W., \& Kraag, G. (1982). The McMaster Health Index Questionnaire as a measure of quality of life for patients with rheumatoid disease. The Journal of Rheumatology, 9(5), 780-784.

Costa, P. S., Santos, N. C., Cunha, P., Cotter, J., \& Sousa, $N$. (2013). The use of multiple correspondence analysis to explore associations between categories of qualitative variables in healthy ageing. Journal of Aging Research, 302163. doi: $10.1155 / 2013 / 302163$

Dazord, A. (1997). [Evaluation of care: Taking into account the patients' quality of life and relational elements]. Recherche en Soins Infirmiers (50), 119-130.

Donovan, J. (1991). Patient education and the consultation: The importance of lay beliefs. Annals of the Rheumatic Diseases, 50 Suppl 3, 418-421.
Herdman, M., Fox-Rushby, J., \& Badia, X. (1997). 'Equivalence' and the translation and adaptation of health-related quality of life questionnaires. Quality of Life Research: An International Journal of Quality of Life Aspects of Treatment, Care and Rehabilitation, 6(3), 237-247.

Higginson, I. J., \& Carr, A. J. (2001). Measuring quality of life: Using quality of life measures in the clinical setting. British Medical Journal, 322(7297), 1297-1300.

International Agency for Research on Cancer \& World Health Organization. (2008). Globocan 2008: Cancer incidence and mortality worldwide in 2008. Lyon: authors.

Jirojanakul, P., Skevington, S. M., \& Hudson, J. (2003). Predicting young children's quality of life. Social Science \& Medicine, 57(7), 12771288.

Joyce, C. R. B., McGee, H. M., \& Oboyle, C. A. (1999). Individual quality of life: Approaches to conceptualisation and assessment. Amsterdam, The Netherlands: Harwood Academic.

Jozefiak, T., Larsson, B., Wichstrom, L., Wallander, J., \& Mattejat, F. (2010). Quality of Life as reported by children and parents: a comparison between students and child psychiatric outpatients. Health and Quality of Life Outcomes, 8, 136. doi: 10.1186/1477-7525-8-136

Molzahn, A. E., Kalfoss, M., Schick Makaroff, K., \& Skevington, S. M. (2011). Comparing the importance of different aspects of quality of life to older adults across diverse cultures. Age and Ageing, 40(2), 192-199. doi: 10.1093/ageing/afq156

O’Connell, K. A., \& Skevington, S. M. (2005). The relevance of spirituality, religion and personal beliefs to health-related quality of life: themes from focus groups in Britain. British Journal of Health Psychology, 10(Pt 3), 379-398.

O’Connell, K. A., \& Skevington, S. M. (2007). To measure or not to measure?: Reviewing the assessment of spirituality and religion in health-related quality of life. Chronic Illness, 3(1), 77-87. 
O’Connell, K. A., \& Skevington, S. M. (2010). Spiritual, religious, and personal beliefs are important and distinctive to assessing quality of life in health: A comparison of theoretical models. British Journal of Health Psychology, 15(Pt 4), 729-748. doi: 10.1348/135910709X479799

Ruta, D. A., Garratt, A. M., Leng, M., Russell, I. T., \& MacDonald, L. M. (1994). A new approach to the measurement of quality of life. The Patient-Generated Index. Medical Care, 32(11), 1109-1126.

Sánchez-Pedraza, R., Ballesteros, M. P., \& Anzola, J. D. (2010). [The concept of quality of life in women suffering from cancer]. Revista de Salud Pública (Bogotá), 12(6), 889-902.

Sánchez, R., Alexander-Sierra, F., \& Oliveros, R. (2012). Relationship between quality of life and clinical status in patients with gastrointestinal cancer. Revista Española de Enfermedades Digestivas, 104(11), 584-591.

Sánchez, R., Ballesteros, M., \& Gómez, A. (2009). Medición de la calidad de vida en ensayos clínicos de pacientes con cáncer: un estudio bibliométrico. Revista Colombiana de Cancerología, 13(1), 29-34.

Seidl, E. M., \& Zannon, C. M. (2004). [Quality of life and health: Conceptual and methodological issues]. Cadernos de Saude Publica, 20(2), 580-588.

Skevington, S. M. (2002). Advancing cross-cultural research on quality of life: observations drawn from the WHOQOL development. World Health Organisation Quality of Life Assessment. Quality of Life Research: An International Journal of Quality of Life Aspects of Treatment, Care and Rehabilitation, 11(2), 135-144.

Skevington, S. M., Day, R., Chisholm, A., \& Trueman, P. (2005). How much do doctors use quality of life information in primary care? Testing the trans-theoretical model of behaviour change. Quality of Life Research: An International Journal of Quality of Life Aspects of Treatment, Care and Rehabilitation, 14(4), 911-922.
Skevington, S. M., Dehner, S., Gillison, F. B., McGrath, E. J., \& Lovell, C. R. (2014). How appropriate is the WHOQOL-BREF for assessing the quality of life of adolescents? Psychology \& Health, 29(3), 297-317. doi: 10.1080/08870446.2013.845668

Skevington, S. M., Gunson, K. S., \& O'Connell, K. A. (2013). Introducing the WHOQOL-SRPB BREF: developing a short-form instrument for assessing spiritual, religious and personal beliefs within quality of life. Quality of Life Research: An International Journal of Quality of Life Aspects of Treatment, Care and Rehabilitation, 22(5), 1073-1083. doi: 10.1007/ s11136-012-0237-0

Skevington, S. M., Sartorius, N., \& Amir, M. (2004). Developing methods for assessing quality of life in different cultural settings: The history of the WHOQOL instruments. Social Psychiatry and Psychiatric Epidemiology, 39(1), 1-8. doi: 10.1007/s00127-004-0700-5

Skrzypczak, M., Czerniak, U., \& Laski, P. (2012). Selected elements of socio-demographic status and lifestyle as factors determining subjective assessment of life in women after mastectomy. Contemporary Oncology, 16(6), 569-575. doi: 10.5114/wo.2012.32492

Slevin, M. L., Plant, H., Lynch, D., Drinkwater, J., \& Gregory, W. M. (1988). Who should measure quality of life, the doctor or the patient? British Journal of Cancer, 57(1), 109-112.

Smith, K. W., Avis, N. E., \& Assmann, S. F. (1999). Distinguishing between quality of life and health status in quality of life research: a meta-analysis. Quality of Life Research: An International Journal of Quality of Life Aspects of Treatment, Care and Rehabilitation, 8(5), 447-459.

Stenner, P. H., Cooper, D., \& Skevington, S. M. (2003). Putting the Q into quality of life; the identification of subjective constructions of health-related quality of life using Q methodology. Social Science \& Medicine, 57(11), 2161-2172. 
Testa, M. A. (2000). Interpretation of quality-of-life outcomes: issues that affect magnitude and meaning. Medical Care, 38(9 Suppl), II166-174.

Vig, E. K., Davenport, N. A., \& Pearlman, R. A. (2002). Good deaths, bad deaths, and preferences for the end of life: A qualitative study of geriatric outpatients. Journal of the American Geriatrics Society, 50(9), 1541-1548.

Wallander, J. L., Schmitt, M., \& Koot, H. M. (2001). Quality of life measurement in children and adolescents: issues, instruments, and applications. Journal of Clinical Psychology, 57(4), 571-585. 